\title{
Interacting Effects of Cognitive Load and Adult Age on the Regularity of Whole-Body Motion During Treadmill Walking
}

\author{
Julius Verrel \\ Max Planck Institute for Human Development
}

Michael Schellenbach

Max Planck Institute for Human Development and University

\author{
Martin Lövdén \\ Max Planck Institute for Human Development \\ and Lund University
}

\author{
Sabine Schaefer and Ulman Lindenberger \\ Max Planck Institute for Human Development
}

\begin{abstract}
We investigated effects of concurrent cognitive task difficulty ( $n$-back) on the regularity of whole-body movements during treadmill walking in women and men from 3 age groups (20-30, 60-70, and 70-80 years old). Using principal component analysis of individual gait patterns, we separated main (regular) from residual (irregular) components of whole-body motion. Proportion of residual variance (RV) was used as an index of gait irregularity. The gait in all age groups became more regular (reduced RV) upon introduction of a simple cognitive task (1-back), relative to walking without a concurrent cognitive task. In contrast, parametrically increasing working memory load from 1-back to 4-back led to age-differential effects, with gait patterns becoming more regular in those 20-30 years old, becoming less regular in those 70-80 years old, and showing no significant effects in those 60-70 years old. Our results support the dual-process account of sensorimotor-cognitive interactions (O. Huxhold, S.-C. Li, F. Schmiedek, and U. Lindenberger, 2006), with age-general effects of internal versus external attentional focus and age-specific effects of resource competition with increasing cognitive task difficulty.
\end{abstract}

Keywords: gait, principal component analysis, dual-tasking, aging, working memory

Correlational studies and dual-task experiments point to aginginduced changes in sensorimotor-cognitive couplings (for review, see Schaefer, Huxhold, \& Lindenberger, 2006). When cognitive and sensorimotor tasks are performed simultaneously, older adults show greater dual-task costs than do young adults in cognitive (Li, Lindenberger, Freund, \& Baltes, 2001), sensorimotor (Huxhold, Li, Schmiedek, \& Linden, 2006), or both domains of functioning (Lindenberger, Marsiske, \& Baltes, 2000). However, the experimental evidence concerning attentional effects on gait patterns is mixed (Lövdén, Schaefer, Pohlmeyer, \& Lindenberger, 2008). We studied the effect of concurrent cognitive task difficulty

Julius Verrel, Sabine Schaefer, and Ulman Lindenberger, Center for Lifespan Psychology, Max Planck Institute for Human Development, Berlin, Germany; Martin Lövdén, Center for Lifespan Psychology, Max Planck Institute for Human Development, Berlin, and Department of Psychology, Lund University, Lund, Sweden; Michael Schellenbach, Center for Lifespan Psychology, Max Planck Institute for Human Development, Berlin, and Institute for Geoinformatics, University of Muenster, Muenster, Germany.

We would like to thank the staff of the Sensorimotor-Cognitive Interactions Project, in particular Gabriele Faust and Anna Pohlmeyer, for help with organization and data collection. Julius Verrel is supported by a scholarship from the International Max Planck Research School LIFE (The Life Course: Evolutionary and Ontogenetic Dynamics).

Correspondence concerning this article should be addressed to Julius Verrel, Center for Lifespan Psychology, Max Planck Institute for Human Development, Lentzeallee 94, Berlin 14195, Germany. E-mail: verrel@mpib-berlin.mpg.de
( $n$-back task) on walking regularity in three age groups (adults $20-30,60-70$, and $70-80$ years old) using a regularity measure, based on principal component analysis (PCA), that captures whole-body coordination.

In the $n$-back task (Dobbs \& Rule, 1989), participants are required to monitor a sequence of items (e.g., digits) presented to them and to respond whenever the current item is identical to the one presented $n$ positions before it. This requires continuous updating of working memory (WM) contents. The parameter $n$ allows manipulation of task difficulty (WM load) within the same paradigm. The $n$-back task was recently used to investigate adult age-related effects of cognitive task difficulty on postural control (Huxhold et al., 2006). In that study, introduction of a simple task (1-back vs. no task) led to decreased postural sway (quantified as center of pressure fluctuations) in both young and older adults. In contrast, when WM load was increased from 1-back to 2-back, older adults swayed more, whereas younger adults did not.

Huxhold et al. (2006) proposed a dual-process model to account for their findings, comprising (a) age-general effects of internal versus external attentional focus and (b) effects due to crossdomain resource competition modulated by age (Huxhold et al., 2006; see also Lövdén et al., 2008). First, with an internal focus of attention (i.e., focus on the movement per se), top-down cognitive control (Miller \& Cohen, 2001; O'Reilly, 2006) may interfere with the self-organizing dynamics of the motor system (e.g., Beilock, Berenthal, McCoy, \& Carr, 2004; Wulf \& Prinz, 2001). Introducing a simple cognitive task attenuates this interference by inducing an external focus of attention. Second, higher levels of cognitive task difficulty may hamper motor control performance through 
cross-domain resource competition (Lindenberger et al., 2000; Lövdén et al., 2008; Schaefer et al., 2006; Woollacott \& Shumway-Cook, 2002). The point at which performance decrements due to resource competition outweigh performance improvements due to external focus is assumed to vary as function of individuals' sensorimotor and cognitive resources. Given that both sensorimotor and cognitive resources decline with advancing age (Lindenberger \& Baltes, 1994), motor control is assumed to be more susceptible to cognitive load as age advances.

The generalizability of the Huxhold et al. (2006) model from postural sway during standing to variability in walking is currently not known. The relevant experimental evidence is mixed, as effects of cognitive load on walking parameters have not always been observed (see Lövdén et al., 2008, for discussion). This lack of consistency may reflect (a) the variety of cognitive tasks used (both with respect to type and difficulty level) and (b) the lack of efficient measures of walking performance. Lövdén et al. (2008) addressed the first point by using the $n$-back paradigm (with $n=$ $1,2,3$, or 4). Consistent with the dual-process account described earlier, gait variability - assessed with several step-related variability measures, such as stride-length or step-width variabilitywas reduced by the introduction of an easy (low-complexity) task (1-back), presumably reflecting a shift from internal to external attentional focus. However, the predicted age-specific increase for increasing WM load from 1-back to 4-back (resource competition) in the older participants was not statistically reliable. A possible explanation for this failure to find the hypothesized Age $\times$ Load interaction refers to dependent variables used in the Lövdén et al. (2008) study, which were restricted to discrete events of the step pattern.

Here, we present a partial reanalysis of the data from Lövdén et al. (2008), introducing an alternative measure of walking regularity based on PCA of individual gait patterns. We predicted that because this approach took into account time-continuous information of whole-body coordination, it would yield a more efficient and valid index of cognitive load-induced changes in whole-body coordination than step-related measures. Using PCA, we split kinematic walking data into a main (regular) pattern and a residual (irregular) pattern. Walking irregularity was quantified by the residual variance, that is, the relative amount of variance in the residual pattern. In addition to the data from Lövdén et al. (2008), we included data from a third age group (60-70 years old). Thus, data from adults of three different age groups (20-30, 60-70, and $70-80$ years) are reported, thereby allowing a more fine-grained analysis of the relation between adult age and attentional-loadinduced decrements in whole-body coordination.

On the basis of a dual-process account proposed by Huxhold et al. (2006), we predicted different effects of adding a simple cognitive task (1-back compared with no load) versus further increasing WM load. More specifically, we hypothesized that gait patterns of participants from all age groups would be more regular in the 1-back compared with the no-load condition, due to an externalization of attentional focus. The effect of increasing WM load from 1-back to 4-back on the regularity of gait patterns was hypothesized to differ by age, so that older participants would show higher dual-task costs than young participants. At the motor level, this age-differential increase in dual-task costs was expected to result in a greater decrease of walking regularity in the older age groups. In accord with the well-documented decline of sensorimo- tor, attentional, and cognitive functions with advancing adult age and within old age (Lindenberger \& Baltes, 1994; Park, Polk, Mikels, Taylor, \& Marshuetz, 2001; Spirduso, Francis, \& MacRae, 2005), the 70- to 80-year-old participants were expected to show the most pronounced effects of WM load on whole-body coordination.

\section{Method}

Part of the raw data (pertaining to the youngest and oldest age groups) was used in a previous study (Lövdén et al., 2008). Only (discrete) step parameters such as step width and stride time were considered in the first analysis. In contrast, continuous motion of the entire body is considered here. Furthermore, data from an additional age group (60-70 years) were included for the present study. Some of the present results (from the 20- to 30-year-old and the 70- to 80-year-old participants) have been presented in abstract form (Verrel, Lövdén, Pohlmeyer, Schaefer, \& Lindenberger, 2007).

\section{Participants}

Ninety-six adults from three age groups (20-30, 60-70, and $70-80$ years old) stratified by sex (i.e., 16 women and 16 men in each age group) were recruited from the participant pool of the Max Planck Institute for Human Development, Berlin, Germany, on the basis of criteria excluding individuals with conditions known to influence balance or gait performance (e.g., Parkinson's disease, diabetes, gout, severe back pain, impaired balance, cardiovascular problems, or artificial hip joint). All participants had normal or corrected-to-normal vision and hearing (according to self-report). To document the age typicality of the sample with respect to cognitive functioning, we assessed perceptual speed (digit-symbol substitution test; Wechsler, 1982) and vocabulary (Mehrfachwahl-Wortschatz-Intelligenztest, MWT-A [MultipleChoice Vocabulary Intelligence Test]; adapted after Lehrl, Merz, Burkard, \& Fischer, 1991).

An analysis of variance (ANOVA) with repeated contrasts for the age group effect revealed that there was a main effect of age group for the digit-symbol substitution test, $F(2,93)=29.59, p<$ .05 , with young adults showing higher performance than the 60 - to 70 -year-olds but with no reliable differences found between the two older age groups (mean score $\pm S D$ for the 20- to 30-yearolds: $64.9 \pm 11.0 ; 60$ - to 70 -year-olds: $47.9 \pm 10.0 ; 70$ - to 80 -year-olds: $46.8 \pm 10.5)$. In the vocabulary test, the main effect of age group was reliable, $F(2,93)=3.56, p=.032$. Follow-up tests indicated that the 60- to 70-year-olds performed above the level of the young adults, whereas differences between the two older age groups were not reliable (mean score $\pm S D$ for the 20to 30 -year-olds: $31.7 \pm 3.1 ; 60$ - to 70 -year-olds: $33.2 \pm 1.9$; 70 to 80 -year-olds: $33.3 \pm 2.8$ ). Thus, we obtained the typical pattern of adult age differences in cognitive performance, with performance decrements for a marker test of perceptual speed belonging to the broad domain of fluid abilities and performance increments for a marker test of verbal knowledge from the broad domain of crystallized abilities (Baltes, 1987; Horn, 1989).

Written consent was obtained from the participants prior to the experiment. Each participant received 50 euros for participation in 
the entire experiment. The ethics committee of the Max Planck Institute for Human Development approved the study.

\section{Apparatus}

We used a 12-camera (infrared V-cam 100 \& 200) motion capture system (Vicon 612, Workstation 4.6; Vicon Ltd., Oxford, UK), sampling at $200 \mathrm{~Hz}$, for recording participants' limb movements while they walked on the treadmill. Reflective markers were placed on relevant anatomical landmarks according to the Vicon PlugInGait model. These included 7 markers on each leg or foot, 4 on the pelvis, 6 on the thorax and shoulder girdle, 6 on each arm or hand, and 4 on the head; amounting to a total of 40 markers. The foot markers were placed on prepared sport shoes; all other markers were applied directly to the skin or attached using straps around wrist and head.

Participants walked on a treadmill (Woodway; Weil am Rhein, Germany), with the walking area $(200 \mathrm{~cm} \times 70 \mathrm{~cm})$ at the level of the surrounding floor. No handrail was present. For safety reasons, a harness was fastened around the waist of the participant and attached to the ceiling. In front of the treadmill, a $200 \mathrm{~cm} \times 270$ $\mathrm{cm}$ screen was mounted. A virtual environment consisting of a straight path was backprojected on the screen. The visual flow of the virtual environment was synchronized to the speed of the treadmill with an empirically established flow-speed ratio.

\section{Design and Procedure}

This study investigated the effect of combining a cognitive task ( $n$-back, with $n=1,2,3$, and 4) with a motor activity (walking) on performance in either of these. Each participant completed two sessions, one involving walking at a fixed speed $(2.5 \mathrm{~km} / \mathrm{hr})$, and the other involving walking at a self-selected speed. The selfselected speed was determined at the beginning of the experimental session and was kept constant across conditions. In all except 3 participants, the fixed speed was slower than the self-selected speed. Also, the deviations between self-selected and fixed speed differed among age groups, with 20- to 30-year-olds walking faster (mean $\pm \mathrm{SD}: 3.72 \pm 0.36 \mathrm{~km} / \mathrm{hr}$ ) compared with the 60 to 70 -year-olds $(3.42 \pm 0.49 \mathrm{~km} / \mathrm{hr})$ and 70 - to 80 -year-olds $(3.11 \pm 0.47 \mathrm{~km} / \mathrm{hr})$. Thus, to assure comparability of the regularity of walking patterns and sensorimotor-cognitive interactions across participants and age groups (Jordan, Challis, $\&$ Newell, 2007), we focused on the self-selected speed condition in the present analysis.

In the $n$-back task, a sequence of 26 digits was presented auditorily via loudspeakers, with interstimulus intervals jittered between $2,850 \mathrm{~ms}$ and 3,150 ms. The task consisted of monitoring this number sequence and indicating whenever the currently presented digit coincided with the one that was given $n$ steps earlier in the sequence. For instance, in the 2-back condition, participants have to respond when the current number is the same as the one presented two steps before. Responses were given verbally ("yes" whenever a target occurred). Trials in all conditions included the same number of targets (six).

We started the session by familiarizing the participants with the treadmill and determining each individual's preferred walking speed in an interactive procedure. The actual experiment was organized in an ABA scheme: In the main experimental condition
(B: dual-tasking), participants performed the 1- to 4-back task while walking on the treadmill. Before and after this (A), control conditions for motor (walking without concurrent cognitive task) and cognitive ( $n$-back task while sitting) performance were presented. In the dual-tasking trials, two instances of each cognitive condition were presented in a row. The order of the cognitive conditions was partially counterbalanced (ascending or descending between 1-back and 4-back). In total, each dual-tasking trial lasted about $90 \mathrm{~s}$, including an initial familiarization phase of $30 \mathrm{~s}$ without cognitive task. Motion capture was restricted to a 20-s interval without targets in order to avoid effects of articulatory movement on the measured walking pattern.

To sum up, the experimental design for the cognitive measures was a 3 (age group: $20-30,60-70$, or $70-80$ years) $\times 2$ (sex) $\times$ 2 (setting: sitting or walking) $\times 4$ (WM load: from 1 -back to 4-back) factorial design. With respect to walking performance, the design was a 3 (age group) $\times 2(\operatorname{sex}) \times 5$ (WM load: no load, from 1-back to 4-back) factorial design.

\section{Data Processing}

Kinematic data from all markers were processed and analyzed separately for each trial and participant according to the following procedure: from the $20 \mathrm{~s}$ recorded in each trial, the middle $15 \mathrm{~s}$ were selected to avoid recording artefacts due to start-and-stop of motion capture and to assure equal registration durations for all participants. Data were represented as Cartesian coordinates in an array of $3,000 \times 120$ dimensions $(15 \mathrm{~s}$ sampled at $200 \mathrm{~Hz}$; three-dimensional information for 40 markers). After correcting for movement relative to the treadmill (e.g., due to temporary lagging behind the constant velocity), the data were submitted to a PCA to split them into main and residual (regular and irregular) components (Daffertshofer, Lamoth, Meijer, \& Beek, 2004). This analysis was performed separately for individual trials. Our measure of gait regularity was the residual variance, defined as the relative amount of variance in the residual pattern (expressed as percentage of total variance), with lower values indicating greater gait regularity. This procedure is described in more detail below.

\section{PCA of Gait Patterns}

PCA makes use of the structure of variability in highdimensional data to produce a lower dimensional approximation on the basis of eigenvalue decomposition of the covariance matrix of the data set. Since the covariance matrix is symmetric, this yields an orthogonal decomposition of data space in onedimensional linear subspaces spanned by the eigenvectors (i.e., the principal components, PCs). The corresponding eigenvalues indicate the amount of variance explained by the PCs. Projecting the original data onto the linear subspace spanned by a certain subset of PCs yields a representation of the data in terms of these PCs. Reducing the data to a certain subset of PCs allows one to apply PCA as a data-driven filter, separating structurally invariant (regular) from variant (irregular) components. For a detailed discussion of PCA applied to kinematic data, we refer to the tutorial by Daffertshofer and colleagues (2004).

Kinematic walking data are highly structured, with two or three PCs typically explaining more than $90 \%$ of the variance. Our analysis confirmed previous results regarding the amount of vari- 
ance explained by the first four PCs, in our case: $98.6 \% \pm 0.58 \%$ (mean $\pm \mathrm{SD}$ ). Also, it has been observed that the coefficient time series of the first four PCs typically consist of highly regular oscillations at the frequency of the stride and step cycle (Daffertshofer et al., 2004; Troje, 2002). Figure 1 illustrates the application of PCA to walking data, showing (in Figure 1a) the first four principal components (visualized here relative to the mean posture; see figure caption) and (in Figure 1b) the corresponding coefficient time series as obtained from the data of one trial of a young participant. Note that PCs were determined separately for each participant and trial. The number of PCs constituting the main pattern was selected globally for all participants, as explained in the following.

There is no universal method for determining the cutoff between regular and irregular patterns in PCA. A frequently used method is to determine discontinuities in the (logarithmic) eigenvalue spectrum (scree plot), as shown in Figure 2. According to this criterion, a cutoff after the first or third PC may seem appropriate. On the other hand, visual inspection of coefficient time series revealed considerable variability between participants and trials. In particular, while regular patterns at stride and step frequency (as shown in Figure 1b) were present in all trials, sometimes they would occur in a different order, or in the fifth or some higher PC. In addition, highly regular higher harmonics, presumably reflecting

a

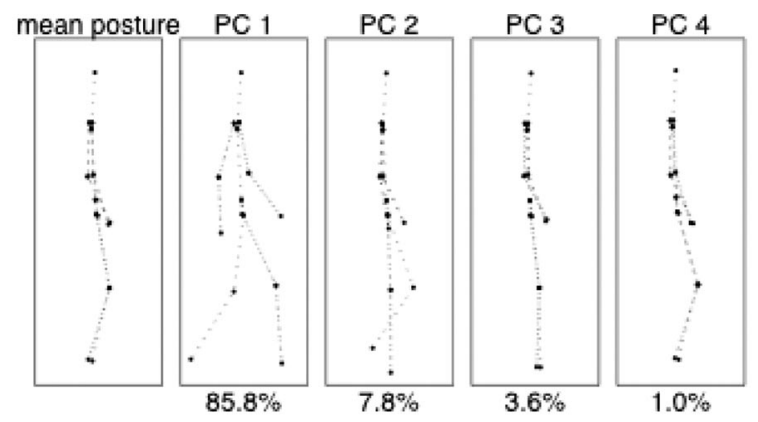

$\mathrm{b}$

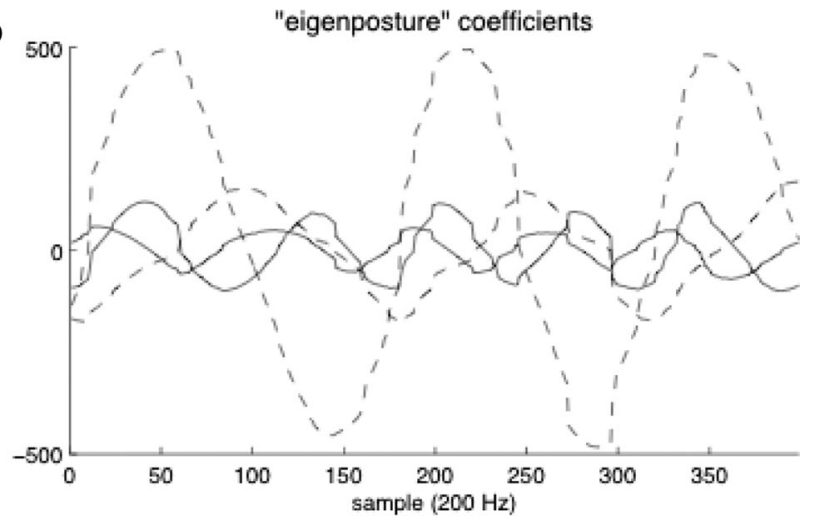

Figure 1. Illustration of principal component analysis (PCA) applied to gait data ( $2 \mathrm{~s}$ from a trial of a young participant). a. Mean posture and first four principal components (PCs), visualized as reconstructed postures. The actual PCs are the deviation between these postures and the mean posture. Percentage of explained variance is given below each PC. b. Coefficient time series of the first four PCs. Coefficients time series of PC 1 and PC 2 (dashed lines) oscillate at stride frequency and PC 3 and PC 4 (solid lines) at step frequency.

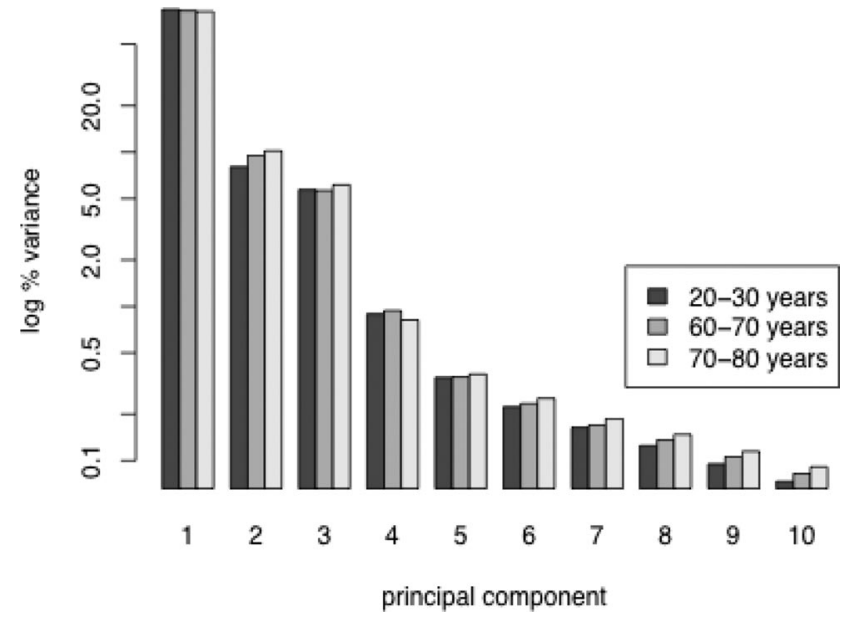

Figure 2. Average eigenvalue spectra (Principal Component [PC] 1-PC $10)$ for the three age groups. Note the logarithmic scale on the vertical axis.

heel strike and toe off, were often observed up to the eighth PC. We therefore chose the first eight PCs as representing the main (regular) pattern. Filtering out this pattern yielded the residual pattern from which our measure of walking regularity-residual variance (RV) — was determined. Precisely, RV was defined as the relative amount of variance (percentage of total variance) present in the residual pattern.

Since the choice of 8 PCs as constituting the main (i.e., regular) pattern is still to some extent arbitrary, RV scores were also computed and plotted for other numbers of PCs (4, 6, and 10). As discussed in the following section, the pattern of results was the same across all four extraction criteria.

\section{Statistical Analysis}

Cognitive and movement performance data from both trials in each condition were averaged. Participants were excluded from further analysis when none of the trials could be analyzed due to missing kinematic data. This was the case for 6 of the 96 participants ( 3 of the 20- to 30-year-olds, 2 of the 60- to 70 -year-olds, and 1 of the 70 - to 80 -year-olds). Since exclusion of participants affected the counterbalancing, we conducted a control analysis with the missing values imputed using linear regression. This analysis produced qualitatively the same results as the original analysis (reported later).

Cognitive performance data (hits - false alarms) were submitted to a 3 (age group: $20-30,60-70$, or $70-80$ years) $\times 2$ (sex) $\times$ 4 (WM load: from 1-back to 4-back) $\times 2$ (setting: sitting or walking) ANOVA with WM load and setting as within-subject factors. Post hoc tests were performed using Welch's two-sample $t$ tests assessing group effects separately at all $n$-back conditions, with significance level Bonferroni adjusted for multiple comparisons.

We analyzed walking performance (RV, for main pattern consisting of eight PCs) using ANOVAs with age group, sex and WM load as between- and within-subject factors. Effects of no-task versus 1-back and of increasing WM load from 1- to 4-back were analyzed separately. The former were analyzed using a 3 (age 
group: $20-30,60-70$, or $70-80$ years $) \times 2($ sex $) \times 2$ (WM load: no-load or 1-back) ANOVA, the latter using a 3 (age group) $\times 2$ $($ sex $) \times 4$ (WM load: from 1- to 4-back) ANOVA. In addition, we computed individual trends for each participant separately as the slopes of RV as a function of increasing WM load (from 1-back to 4-back) using linear regression. To assess age-related effects of parametrically increasing WM load, we compared slopes of the three age groups to zero and to one another using two-tailed one-sample and Welch's two-sample $t$ tests. Significance level was globally set to .05 . Effect sizes are reported as (nonpartial) eta squares.

\section{Results}

Figure 3 shows WM performance as a function of age group, task difficulty ( $n$-back), and setting (sitting vs. walking). Statistics revealed significant main effects of age group, $F(2,84)=14.77$, $p<.05, \eta^{2}=.26$; setting, $F(1,84)=8.48, p<.05, \eta^{2}=.09$; and WM load, $F(3,252)=316.3, p=.05, \eta^{2}=.75$, as well as an Age Group $\times \mathrm{WM}$ Load interaction, $F(6,252)=9.82, p<.05, \eta^{2}=$ .05 . Note that this interaction effect may reflect a ceiling effect for the 1-back condition. Comparison of means revealed that the main effect of setting reflected improved performance in the $n$-back task while participants were walking compared with while they were sitting. Post hoc $t$ tests addressing the interaction of Age Group $\times$ WM Load showed that the age groups did not differ with respect to performance in the 1-back condition. From the 2-back condition onwards, participants of the two older age groups performed below the level of the young adults. No reliable difference was found between the two older age groups.

Walking regularity scores are plotted in Figure 4. For illustrative purposes, we plotted them for four versions of RV based on main patterns consisting of the first 4, 6, 8, and 10 PCs. All of them show the same pattern of decreasing uniformly across age groups from no-load to 1-back, and age-specific increases or decreases for higher WM loads. For reasons discussed earlier, RV-data based on 8 PCs (shown in Figure 4c) were used for statistical analysis.

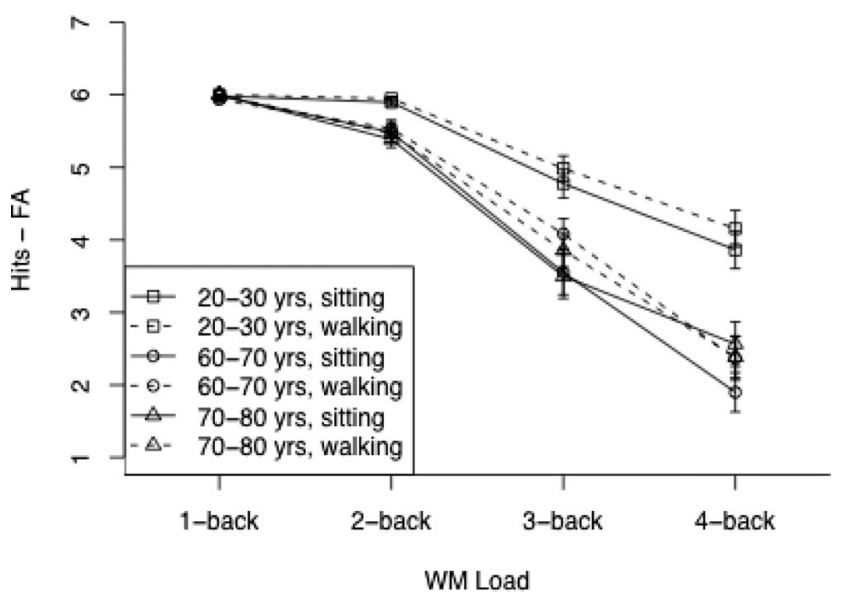

Figure 3. Working memory (WM) performance (hits - false alarms [FA]) as a function of age group, WM load ( $n$-back), and setting (sitting vs. walking). Note that the maximum possible score was six, so participants performed at ceiling in the 1-back and partly the 2-back condition. Error bars represent standard error of the mean.
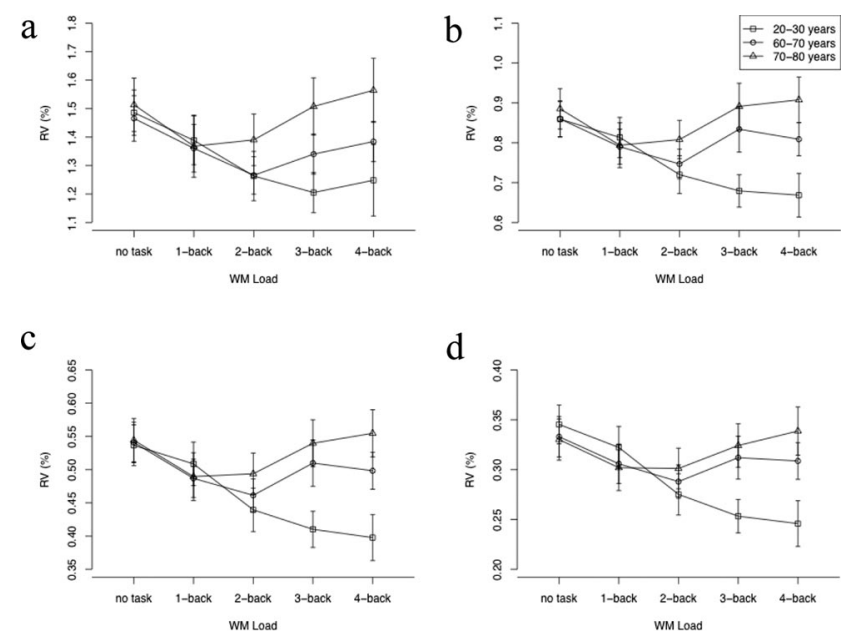

Figure 4. Residual variance (RV) as a function of age group and working memory (WM) load. Results are plotted for RV based on main patterns consisting of the (a) first 4, (b) 6, (c) 8, and (d) 10 principal components. Statistical analysis was performed on the data plotted in Figure 4c. Error bars represent standard error of the mean.

Statistical analysis of RV scores in the no-load versus 1-back condition showed a main effect of WM load, $F(1,84)=14.5, p<$ $.05, \eta^{2}=.14$, with smaller RV (higher regularity) in the 1 -back condition and no further significant effects (for all other effects, $p>.05)$.

The ANOVA addressing the effect of increasing WM load from 1-back to 4-back showed a significant Age Group $\times$ WM Load interaction, $F(6,252)=7.01, p<.05, \eta^{2}=0.14$, and no other reliable effects (all $p>.05$ ). One-sample $t$ tests performed on individual slopes of $\mathrm{RV}$ as a function of WM load indicated negative slopes in the youngest (20-30 years) group, $t(28)=$ $-6.15, p<.05, \eta^{2}=.57$; positive slopes in the oldest $(70-80$ years) group, $t(30)=2.74, p<.05, \eta^{2}=.20$; and no significant deviation from zero in the intermediate $60-70$ years group, $t(29)=$ 1.08. Pairwise comparisons of the groups using two-sample $t$ tests showed that the youngest group differed from both of the older groups: comparison with 60 - to 70-year-olds: $t(53.7)=4.59, p<$ $.05, \eta^{2}=.28$; comparison with 70 - to 80 -year-olds: $t(51.7)=$ 5.70, $p<.05, \eta^{2}=.39$. In contrast, no reliable difference was found between the two groups of older adults, $t(58.2)=-1.35$.

Since the analysis of the number of false alarms occurring during the recording interval (in which no targets of the $n$-back task were presented) showed a similar Age Group $\times$ WM Load interaction as that found for the RV, an ANCOVA with the number of false alarms during movement registration added as a covariate was performed as well. The number of false alarms correlated significantly with $\mathrm{RV}, F(1,251)=15.5, p<.05, \eta^{2}=.05$, but did not eliminate the Age Group $\times$ WM Load interaction, $F(6,251)=$ 5.13, $p<.05, \eta^{2}=.10$.

\section{Discussion}

In this study, we investigated the effect of a concurrent cognitive task on the regularity of whole-body coordination during treadmill walking in three adult age groups, 20-30, 60-70, and 70-80 
years. For participants of all ages, gait patterns became more regular when performing a simple cognitive task (1-back) relative to walking without additional cognitive task, with no reliable group differences in the degree of increase in regularity. In contrast, further increments in cognitive task difficulty (i.e., from 1-back to 2-, 3-, and 4-back) led to age-differential effects: In young adults, gait patterns became more regular; in the oldest age group (70- to 80-year-olds), gait patterns became more irregular; in the group of 60- to 70-year-olds, no reliable trends were found.

With respect to cognitive performance, we found a rather unexpected main effect of task setting (single- vs. dual-task; i.e., sitting vs. walking) on WM performance, indicating a dual-task gain, instead of costs, in the cognitive domain. Schaefer, Lövdén, Wieckhorst, and Lindenberger (2008) found similar effects in young adults (20- to 30-years-old) and children (9 years) using the same paradigm. With respect to aging, Molloy, Beerschoten, Borrie, Crilly, and Cape (1988) found a short physical exercise intervention to have positive effects on a number of scores of cognitive function. The beneficial effect of walking on cognitive performance observed in the present study may be explained by general increases in arousal induced by physical activity (e.g., Adam, Teeken, Ypelaar, Verstappen, \& Paas, 1997). Future studies should address this effect in more detail and over a broader range of cognitive tasks, in particular the question of whether and in what way it may interact with adult age.

Our findings on walking regularity are consistent with the dual-process account of sensorimotor-cognitive interactions proposed by Huxhold et al. (2006). According to this account, cognitive activity at a low difficulty level induces an external focus of attention, allowing automatized and self-organizational mechanisms in the motor system to unfold (e.g., Beilock et al., 2004; Wulf \& Prinz, 2001). This effect is assumed to be relatively age invariant. In contrast, higher difficulty levels are hypothesized to lead to cross-domain resource competition (Lindenberger et al., 2000; Schaefer et al., 2006; Woollacott \& Shumway-Cook, 2002), the degree of which is assumed to differ by cognitive and sensorimotor resources, with the former including attentional mechanisms and WM. Both cognitive and sensorimotor resources are known to decline with advancing age (Lindenberger \& Baltes, 1994; Schaefer et al., 2006). Hence, effects due to resource competition are supposed to become more pronounced with advancing adult age.

The results of this study are consistent with both predictions, thereby extending the generality of the dual-process account from postural sway while standing (e.g., Huxhold et al., 2006) to dualtasked walking. In contrast to an earlier analysis based on steppattern variability (Lövdén et al., 2008), we found reliable dualtask costs in the sensorimotor domain (walking regularity) for high cognitive loads in the oldest age group. At the same time, walking regularity increased with cognitive task difficulty in the young adult group, suggesting that young adults may possess a reserve capacity for enhancing the automaticity of gait when cognitive resources are invested into other domains or, alternatively, that the externalization of attentional focus is a gradual rather than a steplike process. A number of studies have found motor learning and (skilled) performance to be enhanced by an external attentional focus (e.g., Beilock et al., 2004; Wulf \& Prinz, 2001; Wulf $\& \mathrm{Su}, 2007)$. The pattern observed in the group of young participants indicates that it would be worthwhile to explore further the effect of different levels of cognitive task difficulty on motor performance and learning.

To assess walking performance, we used a regularity measure based on PCA of whole-body kinematics. First, PCA was used to split the data into a main (regular) portion and a residual (irregular) portion. Then, the RV (relative amount of variance in the residual portion) was determined as a measure of walking irregularity. Our choice for the splitting between main and residual patterns was guided by the regularity (sinusoidal pattern) of the coefficient time series, which suggested that the main pattern should be defined on the basis of the first 8 PCs. Since this choice was somewhat arbitrary, we also plotted RV scores for other choices (cutting off at PC 4, PC 6, and PC 10). All of these plots show the same overall pattern that we have described, underlining the robustness of our findings.

In the present study, RV displayed several desirable characteristics. First, significant effects could be detected despite a relatively short interval of data collection per condition (not $>30 \mathrm{~s}$ total). Second, in contrast to variability measures based on the step pattern for which older adults typically show higher overall variability (e.g., Lövdén et al., 2008), RV did not show main effects of age group. When present, such main effects limit the interpretability of ordinal interactions (cf. Loftus, 1978). The absence of a main effect of age group in RV may be due to the fact that the main pattern (hence, the filter applied to determine the RV) was individually "tailored" to each participant's biomechanical and movement characteristics. RV was computed by a data-driven filtering procedure (PCA) rather than based on a functional model of walking. To further explore the functional significance of components contributing to walking irregularity, researchers could use PCA in the study of whole-body coordination by examining the factor-loading structure (cf. Lamoth, Daffertshofer, Meijer, \& Beek, 2006, Lamoth et al., 2004, Lamoth, Meijer, Daffertshofer, Wuisman, \& Beek, 2006) or by considering more refined time series analyses of PC coefficients.

\section{References}

Adam, J., Teeken, J., Ypelaar, P., Verstappen, F., \& Paas, F. (1997). Exercise-induced arousal and information processing. International Journal of Sport Psychology, 28, 217-226.

Baltes, P. B. (1987). Theoretical propositions of life-span developmental psychology — on the dynamics between growth and decline. Developmental Psychology, 23, 611-626.

Beilock, S. L., Bertenthal, B. I., McCoy, A. M., \& Carr, T. H. (2004). Haste does not always make waste: Expertise, direction of attention, and speed versus accuracy in performing sensorimotor skills. Psychonomic Bulletin \& Review, 11, 373-379.

Daffertshofer, A., Lamoth, C. J. C., Meijer, O. G., \& Beek, P. J. (2004). PCA in studying coordination and variability: A tutorial. Clinical Biomechanics, 19, 415-428.

Dobbs, A. R., \& Rule, B. G. (1989). Adult age differences in working memory. Psychology and Aging, 4, 500-503.

Horn, J. (1989). Models of intelligence. In Intelligence: Measurement, theory, and public policy. Proceedings of a symposium in honor of Lloyd G. Humphreys, Champaign, IL: University of Illinois Press.

Huxhold, O., Li, S.-C., Schmiedek, F., \& Lindenberger, U. (2006). Dualtasking postural control. aging and the effects of cognitive demand in conjunction with focus of attention. Brain Research Bulletin, 69, 294305. 
Jordan, K., Challis, J. H., \& Newell, K. M. (2007). Walking speed influences on gait cycle variability. Gait Posture, 26, 128-134.

Lamoth, C. J. C., Daffertshofer, A., Meijer, O. G., \& Beek, P. J. (2006). How do persons with chronic low back pain speed up and slow down? Trunk-pelvis coordination and lumbar erector spinae activity during gait. Gait Posture, 23, 230-239.

Lamoth, C. J. C., Daffertshofer, A., Meijer, O. G., Moseley, G. L., Wuisman, P. I. J. M., \& Beek, P. J. (2004). Effects of experimentally induced pain and fear of pain on trunk coordination and back muscle activity during walking. Clinical Biomechanics, 19, 551-563.

Lamoth, C. J. C., Meijer, O. G., Daffertshofer, A., Wuisman, P. I. J. M., \& Beek, P. J. (2006). Effects of chronic low back pain on trunk coordination and back muscle activity during walking: Changes in motor control. European Spine Journal, 15, 23-40.

Lehrl, S., Merz, J., Burkard, G., \& Fischer, B. (1991). Manual zum MWT-A [Manual for MWT-A]. Erlangen, Germany: Perimed.

Li, K. Z., Lindenberger, U., Freund, A. M., \& Baltes, P. B. (2001). Walking while memorizing: Age-related differences in compensatory behavior. Psychological Science, 12, 230-237.

Lindenberger, U., \& Baltes, P. B. (1994). Sensory functioning and intelligence in old age: A strong connection. Psychology and Aging, 9, 339-355.

Lindenberger, U., Marsiske, M., \& Baltes, P. B. (2000). Memorizing while walking: Increase in dual-task costs from young adulthood to old age. Psychology and Aging, 15, 417-436.

Loftus, G. R. (1978). On interpretation of interactions. Memory \& Cognition, 6, 312-319.

Lövdén, M., Schaefer, S., Pohlmeyer, A. E., \& Lindenberger, U. (2008). Walking variability and working memory load in aging: A dual-process account relating cognitive control to motor control performance. Journals of Gerontology, Series B: Psychological Sciences and Social Sciences, 63, 121-128.

Miller, E. K., \& Cohen, J. D. (2001). An integrative theory of prefrontal cortex function. Annual Review of Neuroscience, 24, 167-202.

Molloy, D. W., Beerschoten, D. A., Borrie, M. J., Crilly, R. G., \& Cape, R. D. (1988). Acute effects of exercise on neuropsychological function in elderly subjects. Journal of the American Geriatrics Society, 36, $29-33$.
O'Reilly, R. C. (2006, October 6). Biologically based computational models of high-level cognition. Science, 314, 91-94.

Park, D. C., Polk, T. A., Mikels, J. A., Taylor, S. F., \& Marshuetz, C. (2001). Cerebral aging: Integration of brain and behavioral models of cognitive function. Dialogues in Clinical Neuroscience, 3, 151-166.

Schaefer, S., Huxhold, O., \& Lindenberger, U. (200 6). Healthy mind in healthy body? A review of sensorimotor-cognitive interdependencies in old age. European Review of Aging and Physical Activity, 3, 45-54.

Schaefer, S., Lövdén, M., Wieckhorst, B., \& Lindenberger, U. (2008). Cognitive performance is improved while walking: Differences in cognitive-sensorimotor couplings between children and young adults. Manuscript submitted for publication.

Spirduso, W. W., Francis, K. L., \& MacRae, P. G. (2005). Physical dimensions of aging (2nd ed.). Champaign, IL: Human Kinetics.

Troje, N. F. (2002). Decomposing biological motion: A framework for analysis and synthesis of human gait patterns. Journal of Vision, 2, 371-387.

Verrel, J., Lövdén, M., Pohlmeyer, A., Schaefer, S., \& Lindenberger, U. (2007). Adult age-specific and age-general effects of cognitive load on the regularity of whole-body coordination in dual-tasked walking. In S. Henry, J. Fung, \& J. Jeka (Eds.), Proceedings of the 18th conference of the International Society for Posture and Gait Research. Burlington, VT: International Society for Posture and Gait Research.

Wechsler, D. (1982). Handanweisung zum Wechsler-Intelligenztest für Erwachsene [Manual of the Hamburg-Wechsler Adult Intelligence Tests]. Bern, Switzerland: Huber.

Woollacott, M., \& Shumway-Cook, A. (2002). Attention and the control of posture and gait: A review of an emerging area of research. Gait \& Posture, 16, 1-14.

Wulf, G., \& Prinz, W. (2001). Directing attention to movement effects enhances learning: A review. Psychonomic Bulletin \& Review, 8, 648 660 .

Wulf, G., \& Su, J. (2007). An external focus of attention enhances golf shot accuracy in beginners and experts. Research Quarterly for Exercise and Sport, 78, 384-389.

Received February 26, 2008

Revision received June 23, 2008

Accepted July 19, 2008 